ITP-Budapest 546

UTCCP-P-46

UTHEP-389

September 1998

\title{
The end point of the first-order phase transition of the SU(2) gauge-Higgs model on a four-dimensional isotropic lattice *
}

\author{
Y. Aoki ${ }^{\mathrm{a}, \mathrm{b}}$, F. Csikor ${ }^{\mathrm{c}}$, Z. Fodor ${ }^{\mathrm{c}}$, and A. Ukawa ${ }^{\mathrm{a}, \mathrm{b}}$ \\ ${ }^{a}$ Center for Computational Physics, University of Tsukuba, Ibaraki 305-8577, Japan \\ b'Institute of Physics, University of Tsukuba, Ibaraki 305-8571, Japan \\ ${ }^{\mathrm{c} I n s t i t u t e}$ for Theoretical Physics, Eötvös University, H-1088 Budapest, Hungary
}

We report results of a study of the end point of the electroweak phase transition of the SU(2) gauge-Higgs model defined on a four-dimensional isotropic lattice with $N_{t}=2$. Finite-size scaling study of Lee-Yang zeros yields $\lambda_{c}=0.00116(16)$ for the end point. Combined with a zero-temperature measurement of Higgs and $W$ boson masses, this leads to $M_{H, c}=68.2 \pm 6.6 \mathrm{GeV}$ for the critical Higgs boson mass. An independent analysis of Binder cumulant gives a consistent value $\lambda_{c}=0.00102(3)$ for the end point.

\section{Introduction}

Recently study of the end point of the firstorder electroweak phase transition has been pursued [1] since it provides a basic information on the feasibility of electroweak baryogenesis. For the $\mathrm{SU}(2)$ gauge-Higgs model, detailed results have been reported within the three-dimensional effective theory approach [2-5] including estimates of the critical Higgs boson mass at the end point [3 5]. Four-dimensional simulations, employing space-time anisotropic lattices $[6]$ to overcome a multi-scale problem near the end point, have also been carried out [7].

In this article we report on results of our study of the end point using four-dimensional spacetime symmetric lattices, building upon a previous work [8]. While computationally demanding, this approach is conceptually straightforward, whose results provide an independent check on those obtained with other methods.

\section{Finite-Temperature Simulation}

We work with the standard $\mathrm{SU}(2)$ gauge-Higgs model Lagrangian given by

$$
S=S_{g}+\sum_{x}\left\{\sum_{\mu} 2 \kappa L_{x, \mu}-\rho_{x}^{2}-\lambda\left(\rho_{x}^{2}-1\right)^{2}\right\},
$$

\footnotetext{
*Talk presented by Y. Aoki at Lattice 98, Boulder, USA.
}

$L_{x, \mu} \equiv \frac{1}{2} \operatorname{Tr}\left(\Phi_{x}^{\dagger} U_{x, \mu} \Phi_{x+\hat{\mu}}\right), \rho_{x}^{2} \equiv \frac{1}{2} \operatorname{Tr}\left(\Phi_{x}^{\dagger} \Phi_{x}\right)$,

where $S_{g}$ is the plaquette gauge action with the gauge coupling $\beta=4 / g^{2}$. This work is made for the temporal extension $N_{t}=2$. For spatial lattices we employ $N_{s}^{3}=20^{3}, 24^{3}, 32^{3}, 40^{3}, 50^{3}$ and $60^{3}$. We set the gauge coupling $\beta=8$, and make simulations at five values of the scalar selfcoupling $\lambda=0.00075,0.001,0.00135,0.00145$ and 0.0017235 , which cover the range of Higgs boson mass given by $57 \lesssim M_{H} \lesssim 85 \mathrm{GeV}$ [8]. The Higgs field hopping parameter $\kappa$ is tuned to the vicinity of the pseudo critical point. For each parameter point at least $10^{5}$ iterations of hybrid overrelaxation are made. For further technical details we refer to Ref. 8 .

\section{Analysis of Lee-Yang Zeros}

We consider Lee-Yang zeros of the partition function on the complex $\kappa$-plane at fixed $\lambda$. To search for the zeros we carry out reweighting in $\kappa$ in both imaginary and real directions. In Fig. 1 we show the absolute value of the partition function normalized by its value at the real axis,

$Z_{\text {norm }} \equiv|Z(\operatorname{Re} \kappa, \operatorname{Im} \kappa) / Z(\operatorname{Re} \kappa, 0)|$

measured at $\lambda=0.00075$ and $N_{s}=60$. We observe three zeros in this case. 


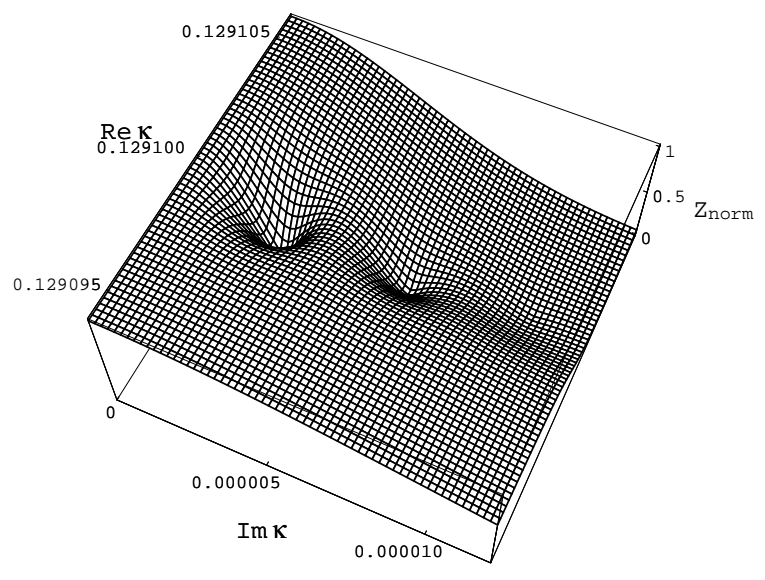

Figure 1. Absolute value of normalized partition function as a function of complex $\kappa$ for $\lambda=0.00075$ and $N_{s}=60$.

Let us call the zero nearest the real axis as first zero, and denote its location by $\kappa_{0}$. The value of $\kappa_{0}$ depends on spatial volume $V=N_{s}^{3}$ as well as on $\lambda$. The first-order phase transition of the model terminates when the infinite volume limit $\kappa_{0}(V \rightarrow \infty)$ deviates away from the real axis.

We show in Fig. 2 all of our results for the imaginary part of the first zero $\operatorname{Im} \kappa_{0}(V)$ as a function of volume. The infinite volume values are extracted by a $\chi^{2}$ fitting employing a scaling ansatz $\operatorname{Im} \kappa_{0}(V)=\kappa_{0}^{c}+C V^{-\nu}$.

Fit results for $\kappa_{0}^{c}$ are shown with filled symbols in Fig. 3. Open symbols are obtained by reweighting the partition function in the variable $\lambda$, starting from histogram results corresponding to the filled symbol of the same shape, and recalculating the first zero. The agreement of open symbols of different shapes within errors shows that reweighting from different values of $\lambda$ gives consistent results in the middle.

At small couplings $\lambda \lesssim 0.001, \kappa_{0}^{c}$ is consistent with zero, which agrees with the result of Ref. [8] that the transition is of first order in this region. At large couplings $\lambda \gtrsim 0.0013, \kappa_{0}^{c}$ no longer vanishes, and hence there is no phase transition. In order to determine the end point of the phase transition, we take the three filled points at $\lambda=0.00135,0.00145$ and 0.0017235 directly

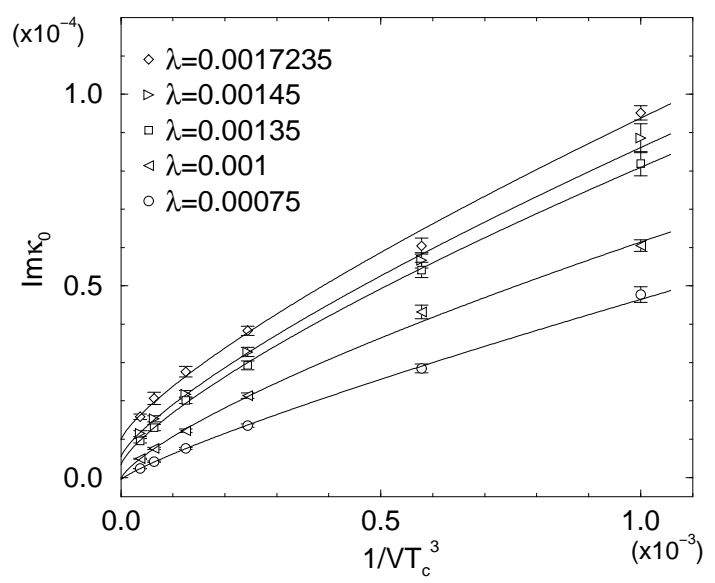

Figure 2. Imaginary part of first Lee-Yang zero as a function of inverse volume normalized by the critical temperature. Solid lines are least $\chi^{2}$ fits with $\operatorname{Im} \kappa_{0}(V)=\kappa_{0}^{c}+C V^{-\nu}$.

obtained from independent simulations without $\lambda$-reweighting, and make a fit with a function linear in $\lambda$. This gives the position of the end point to be $\lambda_{c}=0.00116(16)$.

\section{Binder Cumulant}

The Binder cumulant we study is defined in terms of $L_{s}=\sum_{x, \mu=1,2,3} L_{x, \mu} / 3 V N_{t}$ through

$B_{L_{s}}(\kappa) \equiv 1-\left\langle L_{s}^{4}\right\rangle / 3\left\langle L_{s}^{2}\right\rangle^{2}$.

We evaluate the minimum of the cumulant as a function of $\kappa$ for a given $\lambda$ and volume. We then use a scaling ansatz, $B_{L_{s}}^{\min }=B_{L_{s}}^{c}+C V^{-\nu}$, to extract the infinite-volume value.

In Fig. 1 we show $-\left(B_{L_{s}}^{c}-2 / 3\right)$ as a function of $\lambda$, where meaning of symbols are the same as in Fig. 3. A change of behavior from non-vanishing values to those consistent with zero at $\lambda \approx 0.001$ shows that the first-order phase transition terminates around this value. Linearly extrapolating the two independent data at $\lambda=0.00075$ and 0.001 yields $\lambda_{c}=0.00102(3)$ for the end point, which is consistent with the result from our study of Lee-Yang zeros. 


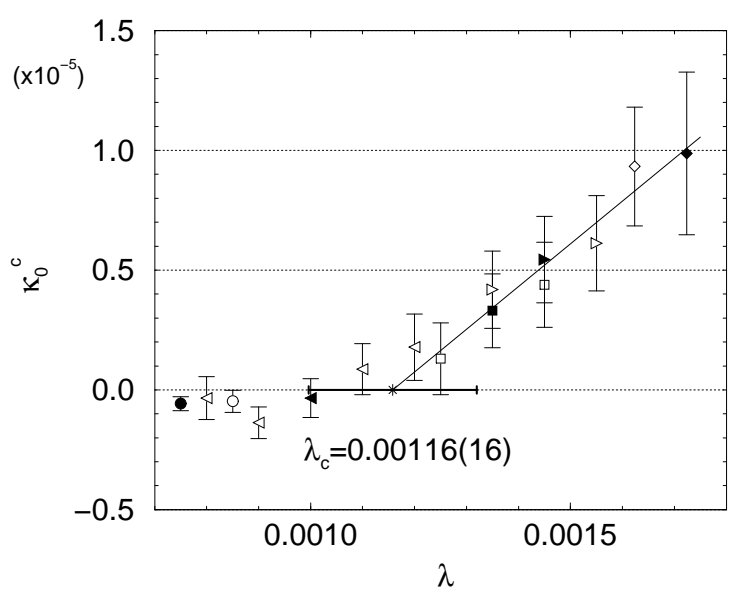

Figure 3. Imaginary part of first Lee-Yang zero at infinite-volume limit as a function of Higgs self coupling. Solid line is a linear fit to $\lambda=$ $0.00135,0.00145$ and 0.0017235 (filled symbols).

\section{Critical Higgs Boson Mass}

To estimate the critical mass $M_{H, c}$ of the Higgs boson at the end point, we carry out a zerotemperature simulation following the method of Ref. [9]. Mass measurements are made at two points given by $\left(\lambda, \kappa=\kappa_{c}\left(\lambda, N_{t}=2\right)\right)$ for $\lambda=$ 0.0011 and 0.00125 employing several lattice sizes to control finite-volume effect. Our preliminary results are $M_{H}=65.8 \pm 0.6 \mathrm{GeV}(\lambda=0.0011)$ and $M_{H}=72.0 \pm 0.4 \mathrm{GeV}(\lambda=0.00125)$. Interpolating to the critical value $\lambda_{c}=0.00116(16)$ from the Lee-Yang zero analysis, we find $M_{H, c}=$ $68.2 \pm 6.6 \mathrm{GeV}$ using $M_{W}=80 \mathrm{GeV}$ as input. The error is dominated by that of $\lambda_{c}$.

\section{Conclusions}

Our result for the critical Higgs boson mass for $N_{t}=2$ is in agreement with the value $M_{H, c}=$ $74.6 \pm 0.9 \mathrm{GeV}$ [7] obtained in a four-dimensional anisotropic lattice simulation for the same temporal size. The same work also reported that the critical mass decreases for larger temporal size, and extrapolates to $M_{H, c}=66.5 \pm 1.4 \mathrm{GeV}$ in the continuum limit. This value is consistent with the three-dimensional result $67.0 \pm 0.8 \mathrm{GeV}$ 泪. Thus results from various methods converge well, and

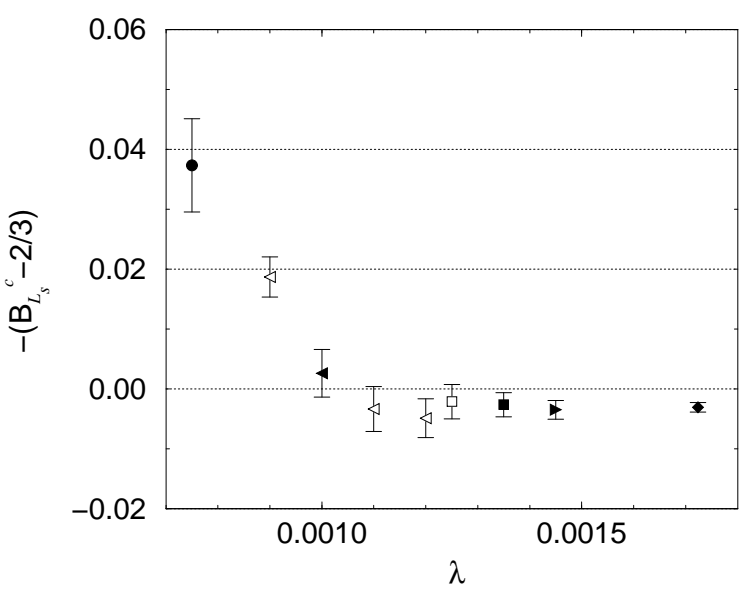

Figure 4. Minimum value of Binder cumulant of $L_{s}$ at infinite volume limit as a function of $\lambda$.

indicate that electroweak baryogenesis is unlikely within the Minimal Standard Model.

Part of this work was carried out while ZF was visiting KEK by the Foreign Researcher Program of the Ministry of Education. This work is supported in part by Grants-in-Aid of the Ministry of Education (No. 10640246).

\section{REFERENCES}

1. M. Laine, in these proceedings.

2. K. Kajantie, M. Laine, K. Rummukainen, and M. Shaposhnikov, Phys. Rev. Lett. 77 (1996) 2887; Nucl. Phys. B493 (1997) 413.

3. F. Karsch, T. Neuhaus, A. Patkós and J. Rank, Nucl. Phys. B (Proc. Suppl.) 53 (1997) 623.

4. M. Gürtler, E.-M. Ilgenfritz, and A. Schiller, Phys. Rev. D56 (1997) 3888.

5. K. Rummukainen, M. Tsypin, K. Kajantie, M. Laine, and M. Shaposhnikov, heplat/9805013.

6. F. Csikor, Z. Fodor, and J. Heitger, heplat/9804026.

7. F. Csikor, Z. Fodor, and J. Heitger, hepph/9809291.

8. Y. Aoki, Phys. Rev. D 56 (1997) 3860.

9. F. Csikor, Z. Fodor, J. Hein, A. Jaster, and I. Montvay, Nucl. Phys. B474 (1996) 421. 